\title{
Synchronized Time-Lens Source for Coherent Raman Scattering Microscopy
}

\section{Citation}

Wang, Ke, Christian W. Freudiger, Jennifer H. Lee, Brian G. Saar, X. Sunney Xie, and Chris Xu. 2010. Synchronized time-lens source for coherent raman scattering microscopy. Optics Express 18(23): 24019-24024.

\section{Published Version}

doi:10.1364/OE.18.024019

\section{Permanent link}

http://nrs.harvard.edu/urn-3:HUL.InstRepos:10419413

\section{Terms of Use}

This article was downloaded from Harvard University's DASH repository, and is made available under the terms and conditions applicable to Open Access Policy Articles, as set forth at http:// nrs.harvard.edu/urn-3:HUL.InstRepos:dash.current.terms-of-use\#OAP

\section{Share Your Story}

The Harvard community has made this article openly available.

Please share how this access benefits you. Submit a story.

Accessibility 


\title{
Synchronized time-lens source for coherent Raman scattering microscopy
}

\author{
Ke Wang, ${ }^{1, *}$ Christian W. Freudiger, ${ }^{2,3}$ Jennifer H. Lee, ${ }^{1}$ Brian G. Saar, ${ }^{2}$ X. Sunney Xie ${ }^{2}$ \\ and Chris $\mathrm{Xu}^{1}$ \\ ${ }^{1}$ School of Applied and Engineering Physics, Cornell University, Ithaca, New York 14853, USA \\ ${ }^{2}$ Department of Chemistry and Chemical Biology, Harvard University, Cambridge, Massachusetts 02138, USA \\ ${ }^{3}$ Department of Physics, Harvard University, Cambridge, Massachusetts 02138, USA \\ *kw376@cornell.edu
}

\begin{abstract}
We use the time-lens concept to demonstrate a new scheme for synchronization of two pulsed light sources for biological imaging. An all fiber, $1064 \mathrm{~nm}$ time-lens source is synchronized to a picosecond solid-state Ti: Sapphire mode-locked laser by using the mode-locked laser pulses as the clock. We demonstrate the application of this synchronized source for CARS and SRS imaging by imaging mouse tissues. Synchronized two wavelength pulsed source is an important technical difficulty for CARS and SRS imaging. The time-lens source demonstrated here may provide an all fiber, user friendly alternative for future SRS imaging.
\end{abstract}

(C)2010 Optical Society of America

OCIS codes: (320.7090) Ultrafast lasers; (060.2380) Fiber optics sources; (180.5655) Raman microscopy.

\section{References and links}

1. A. Zumbusch, G. R. Holtom, and X. S. Xie, "Three-dimensional vibrational imaging by coherent anti-Stokes Raman scattering," Phys. Rev. Lett. 82(20), 4142 (1999).

2. C. W. Freudiger, W. Min, B. G. Saar, S. Lu, G. R. Holtom, C. He, J. C. Tsai, J. X. Kang, and X. S. Xie, "Labelfree biomedical imaging with high sensitivity by stimulated Raman scattering microscopy," Science 322(5909), 1857-1861 (2008).

3. C. L. Evans, E. O. Potma, M. Puoris'haag, D. Côté, C. P. Lin, and X. S. Xie, "Chemical imaging of tissue in vivo with video-rate coherent anti-Stokes Raman scattering microscopy," Proc. Natl. Acad. Sci. U.S.A. 102(46), $16807-16812(2005)$

4. C. L. Evans, X. Xu, S. Kesari, X. S. Xie, S. T. C. Wong, and G. S. Young, "Chemically-selective imaging of brain structures with CARS microscopy," Opt. Express 15(19), 12076-12087 (2007).

5. T. Baldacchini, and R. Zadoyan, "In situ and real time monitoring of two-photon polymerization using broadband coherent anti-Stokes Raman scattering microscopy," Opt. Express 18(18), 19219-19231 (2010).

6. F. Svedberg, C. Brackmann, T. Hellerer, and A. J. Enejder, "Nonlinear microscopy with fiber laser continuum excitation,”J. Biomed. Opt. 15(2), 026026 (2010).

7. A. F. Pegoraro, A. Ridsdale, D. J. Moffatt, J. P. Pezacki, B. K. Thomas, L. Fu, L. Dong, M. E. Fermann, and A. Stolow, “All-fiber CARS microscopy of live cells," Opt. Express 17(23), 20700-20706 (2009).

8. G. Krauss, T. Hanke, A. Sell, D. Träutlein, A. Leitenstorfer, R. Selm, M. Winterhalder, and A. Zumbusch, "Compact coherent anti-Stokes Raman scattering microscope based on a picosecond two-color Er:fiber laser system,” Opt. Lett. 34(18), 2847-2849 (2009).

9. B. H. Kolner, "Active pulse-compression using an integrated electro-optic phase modulator," Appl. Phys. Lett. 52(14), 1122-1124 (1988).

10. D. H. Broaddus, M. A. Foster, O. Kuzucu, A. C. Turner-Foster, K. W. Koch, M. Lipson, and A. L. Gaeta, "Temporal-imaging system with simple external-clock triggering," Opt. Express 18(13), 14262-14269 (2010).

11. Z. Jiang, D. E. Leaird, and A. M. Weiner, "Optical processing based on spectral line-by-line pulse shaping on a phase-modulated CW laser," IEEE J. Quantum Electron. 42(7), 657-665 (2006).

12. J. van Howe, J. Hansryd, and C. Xu, "Multiwavelength pulse generator using time-lens compression," Opt. Lett. 29(13), 1470-1472 (2004).

13. J. van Howe, J. H. Lee, and C. Xu, "Generation of $3.5 \mathrm{~nJ}$ femtosecond pulses from a continuous-wave laser without mode locking," Opt. Lett. 32(11), 1408-1410 (2007).

14. E. O. Potma, D. J. Jones, J. X. Cheng, X. S. Xie, and J. Ye, "High-sensitivity coherent anti-Stokes Raman scattering microscopy with two tightly synchronized picosecond lasers," Opt. Lett. 27(13), 1168-1170 (2002).

15. W. Min, S. Lu, S. Chong, R. Roy, G. R. Holtom, and X. S. Xie, "Imaging chromophores with undetectable fluorescence by stimulated emission microscopy," Nature 461(7267), 1105-1109 (2009).

16. D. Fu, T. Ye, T. E. Matthews, B. J. Chen, G. Yurtserver, and W. S. Warren, "High-resolution in vivo imaging of blood vessels without labeling," Opt. Lett. 32(18), 2641-2643 (2007). 
17. D. Fu, T. Ye, T. E. Matthews, G. Yurtsever, and W. S. Warren, "Two-color, two-photon, and excited-state absorption microscopy," J. Biomed. Opt. 12(5), 054004 (2007).

\section{Introduction}

Coherent Raman Scattering (CRS) microscopy, with contrast from coherent anti-Stokes Raman scattering (CARS) [1] or stimulated Raman scattering (SRS) [2], allows label-free imaging of biological samples with endogenous image contrast based on vibrational spectroscopy. Imaging with high spatial resolution and speed up to video-rate were demonstrated previously [3]. Recently, SRS has superseded CARS as a contrast mechanism for microscopy, because it has improved sensitivity, no image artifacts from spectral distortions or coherent signal addition, and a linear concentration dependence. There has also been increasing interest in using CRS microscopy for medical diagnostics [4], as well as practical applications outside the field of biological and medical applications, such as studies of two-photon polymerization reaction and mechanical properties of polymer microstructures [5].

An important technical challenge in CRS microscopy is the requirement of two synchronized picosecond excitation sources. For practical CRS applications, the light source must fulfill the following requirements: (1) It must provide for two-color excitation, with at least one color tunable so that the difference frequency matches a molecular vibrational frequency. (2) The two colors must be temporally overlapped, with relative timing jitter much less than the pulse widths. (3) The spectral bandwidth of each color must be narrower than the bandwidth of Raman-active vibrational resonances. A transform-limited pulse width of several picoseconds is typically ideal. (4) Sufficient and comparable average power must be available in both beams.

Current excitation sources for CRS include two mode-locked lasers with a phase-lockedloop (PLL) and fine cavity adjustments to synchronize the pulses, optical parametric oscillators (OPOs) synchronously pumped by mode-locked lasers, and two-color sources based on fiber lasers and continuum generation [6] or subsequent soliton self-frequency shift (SSFS) [7,8]. Synchronized mode-locked lasers and OPOs are bulky, costly, and environmentally sensitive, while previous fiber-based sources either have limited spectral resolution due to the broad spectral bandwidth of femtosecond pulse [6,7] or suffer from low output power [8].

Time-lenses were used in active pulse compression [9], telecommunications [10], and pulse shaping [11]. A time-lens imposes a temporal quadratic phase modulation onto the incident light, analogous to a spatial lens imposing a spatial quadratic phase onto a wavefront in space. The phase modulation broadens the spectrum of the input light, and generates the necessary spectral bandwidth for a short pulse. In practice, the quadratic phase modulation is approximated by applying a sinusoidal drive voltage to an electro-optic (EO) phase modulator. With proper dispersion compensation, picosecond or even femtosecond pulses can be generated from a CW laser $[12,13]$. While the repetition rate of a conventional modelocked laser is constrained by the cavity length, the repetition rate of a time-lens source is entirely determined by the electrical drive signal. Thus, a time-lens source has the remarkable capability of synchronizing to any mode-locked laser.

In this paper, we demonstrate a new scheme for synchronizing two pulsed sources for CRS. We synchronize a time-lens source to a tunable Titanium:Sapphire (Ti:Sa) mode-locked laser. The 1.7-ps, 240-mW synchronized pulse train from the compact, all-fiber time-lens source is well suited for CRS microscopy. In addition, electronic radio frequency (RF) delay is used to adjust the relative time delay between the two pulse trains for temporal overlap. By eliminating mechanical optical delay lines, the time delay of the excitation pulses can be adjusted over a large range without any perturbation of the spatial alignment.

\section{Experimental setup}

The experimental setup of the time-lens source synchronized to a Ti:Sa mode-locked laser (Mira 900, Coherent) is shown in Fig. 1 (dotted box). The GaAs photodetector (ET-4000, 
EOT) converts the 76-MHz, 2-ps optical pulse train into an RF pulse train, which is then divided into two branches. One branch is amplified with broadband RF amplifiers to drive the Mach-Zehnder modulator (MZ1, EOSPACE), which carves a synchronized 76-MHz optical pulse train out of a CW laser (QFBGLD-1060-40, QPhotonics). Due to the limited bandwidth of the components, the intensity-modulated optical pulse has a pulse width of $70 \mathrm{ps}$. The other branch is filtered by a narrowband RF filter centered at $9.95 \mathrm{GHz}(131 \mathrm{st}$ tone of the fundamental $76-\mathrm{MHz}$ repetition rate) with a $3 \mathrm{~dB}$ bandwidth of $50 \mathrm{MHz}$. The resulting 9.95 GHz sinusoid is then amplified to drive the two EO phase modulators (2PMs, EOSPACE) that form the time-lens. The intensity modulator (MZ2) is driven by a $10-\mathrm{MHz}$ square wave to modulate the optical pulses for lock-in detection of the SRS signal. MZ2 replaces the external free-space modulation used in previous experiments [2]. In addition, MZ1 and MZ2 may be combined in one MZ modulator by using an appropriate RF combiner. Two $\mathrm{Yb}^{3+}$-doped fiber amplifiers (preamps) compensate for the optical loss, and the $\mathrm{Yb}^{3+}$ doped fiber power amplifier boosts the output power to $1 \mathrm{~W}$. Dispersion compensation is necessary to compress the chirped 70-ps pulses generated by the time-lens. A fiber circulator and a chirped fiber Bragg grating (CFBG) were used as an all-fiber dispersion compensator. After the dispersion compensation stage, the time-lens source has an output power of 240 $\mathrm{mW}$. Because the time-lens generates a highly chirped pulse with long pulse width (70 ps), it can be immediately amplified without the detrimental nonlinear effects of direct amplification of an ultrashort pulse. The time-lens source is analogous to a fiber-based chirped pulse amplification system but without the initial stretcher, making our system more compact and efficient.

The compressed pulse from the time-lens source is combined with the Ti:Sa laser for CRS imaging. Temporal overlap of the two pulse trains is accomplished through tuning of the RF delay (RF delay 1), consisting of a discrete RF delay (PDL-10A, Colby Instruments) and a continuously tunable RF delay for fine adjustment. The time delay of the excitation pulses can be adjusted over a large range $(0.795 \mathrm{~ns})$ without any mechanical optical delay line, greatly facilitating the spatial and temporal alignment of the excitation beams for CRS. All components of the time-lens source, e.g., the modulators, the fiber amplifiers, and the RF components, are made with mature technologies for fiber-optic communications, ensuring a compact setup and robust operation.

For CRS microscopy, collinear pump- and Stokes-beams are coupled into one of two modified laser scanning upright microscopes (BX61WI/FV300, Olympus for SRS imaging and TCS-SP5, Leica for video-rate CARS imaging). We use a 60x 1.2NA water immersion objective (UPlanApo / IR, Olympus) to focus the beams into a common focal spot in the sample. The beam-size is matched to fill the back-aperture of the objective. For SRS, light is collected in transmission with a 60x 1.4NA oil condenser (Nikon), which is aligned with white-light transmission from a lamp. A telescope is used to image the scanning mirrors onto the photodiode to avoid beam-movement due to laser scanning. A high OD band-pass filter (Chroma Technology, CARS 890/220m) is used to block the Stokes beam and transmit the pump-beam only. A large-area photodiode (FDS1010, Thorlabs) with a $64 \mathrm{~V}$ reverse bias is used for detection of the pump beam. The output current is bandpass-filtered (BBP-10.7, Mini-Circuits) to suppress the high frequency signal due to the laser pulsing (76 MHz) and the low frequency signal fluctuations from scanning the beam through a sample with varying transmission. A high-frequency lock-in amplifier (SR844RF, Stanford Research Systems) is used to demodulate the pump-intensity. The analog X-output of the lock-in amplifier is fed into a modified input of the microscope A/D-converter. For CARS, the signal is collected in the backward direction using the excitation objective lens. A dichroic mirror (770dcxr, Chroma Technology) is used to separate the anti-Stokes signal from the incoming beams. The light is filtered to remove residual pump light using a short pass filter (ET750sp-2p8, Chroma Technology) and detected using the microscope's internal non-descanned photomultiplier tube, whose output is digitized and displayed by the microscope software (LAS AF, Leica Microsystems).

\#136585 - \$15.00 USD Received 13 Oct 2010; revised 27 Oct 2010; accepted 28 Oct 2010; published 2 Nov 2010 (C) 2010 OSA 8 November 2010 / Vol. 18, No. 23 / OPTICS EXPRESS 24021 


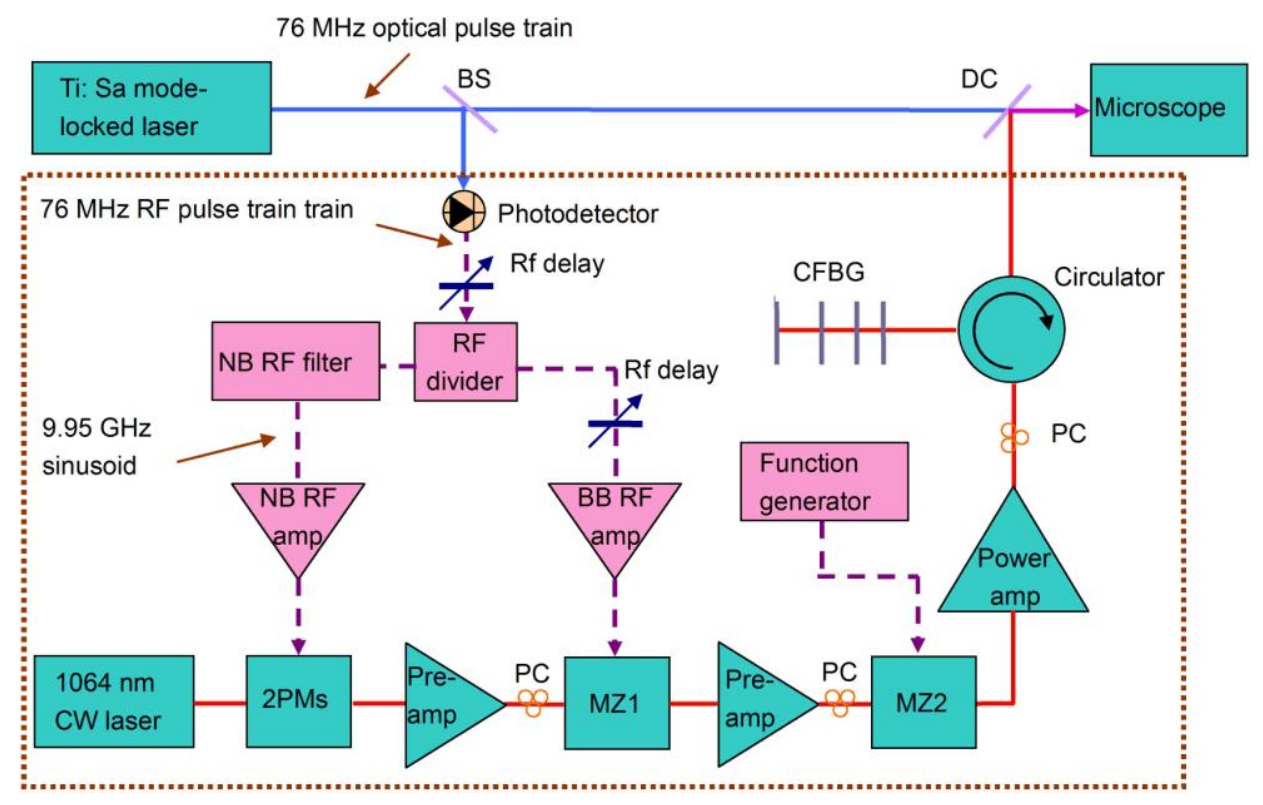

Fig. 1. Experimental setup of the $1064 \mathrm{~nm}$ time-lens source synchronized to a mode-locked laser. The time-lens source (enclosed by the dotted box) consists of a $1064 \mathrm{~nm} \mathrm{CW}$ laser diode, 2 phase modulators (2PMs), 2 Mach-Zehnder intensity modulators (MZ1 and MZ2), a fiber circulator, a chirped fiber Bragg grating (CFBG), and $\mathrm{Yb}^{3+}$-doped fiber amplifiers (YDFAs, 2 preamps and 1 power amp). All fiber devices are polarization maintained except for the YDFAs where polarization controllers (PCs) are used to align the polarization states. The RF signals used to drive the 2 PMs and MZ1 are derived from the fast photodetector. MZ2 is driven with an external function generator at $10 \mathrm{MHz}$ for SRS imaging. BS: beam splitter, DC: dichroic mirror, NB: narrow band, BB: broad band. Electrical paths are labeled with dashed lines, and optical paths are labeled with solid lines.

\section{Experimental results}

We first characterize the time-lens output. The time-lens source has an output spectrum of $1.6 \mathrm{~nm}$ (FWHM, Fig. 2a). Spectral pedestals are due to the small spectral broadening in the fiber pigtail after the CFBG, where the pulse is already compressed. To directly measure the temporal profile of the pulses from the time-lens source, we synchronize the time-lens source to a 130 -fs fiber-based mode-locked laser (Femtolite F100, IMRA), and obtain the crosscorrelation trace. The femtosecond mode-locked laser is centered at $807 \mathrm{~nm}$, and has a repetition rate of $77 \mathrm{MHz}$, the 129th tone of which is $9.95 \mathrm{GHz}$. For the cross-correlation measurement, we used a beta barium borate ( $\beta$-BBO) crystal $(0.5 \mathrm{~mm}$ thick) for collinear sum frequency generation. The sum frequency signal $(459 \mathrm{~nm})$ is filtered by a color filter and measured with a silicon photodiode. We scan the RF delay line, which tunes the relative delay between the time-lens source and the fiber laser, and record the cross-correlation trace with a real-time oscilloscope (Fig. 2b). The pulse width of the time-lens source is $1.7 \mathrm{ps}$. To measure the timing jitter, we delay the femtosecond pulse by half the pulse width of the time-lens source, and record the sum frequency intensity fluctuation at the half maximum (i.e., the location of the steepest slope) of the cross-correlation trace as a function of time [14], also shown in Fig. 2b. Root-mean-square (RMS) jitter of 50 fs was measured over a time span of 350 seconds (sampled at $1 \mathrm{KHz}$ ). The side lobes in the temporal profile (Fig. 2b) are due to uncompensated chirp introduced by the sinusoidal phase modulation [12]. These side lobes, if necessary, can be reduced by phase correction or higher order dispersion compensation $[11,12]$. We followed the same procedure for the cross-correlation and timing jitter measurement between the time-lens source and the picosecond Ti:Sa mode-locked laser. Figure 2c shows the cross-correlation trace with the picosecond Ti:Sa laser, with a FWHM of 
2.9 ps before deconvolution. The measured RMS timing jitter using the sum-frequency generation technique is $120 \mathrm{fs}$ over a measurement time of 350 seconds. For SRS imaging, the time-lens output was modulated by a $10-\mathrm{MHz}$ square wave (Fig. 2d). Synchronization of modulation frequency and laser repetition rate is not necessary [2].

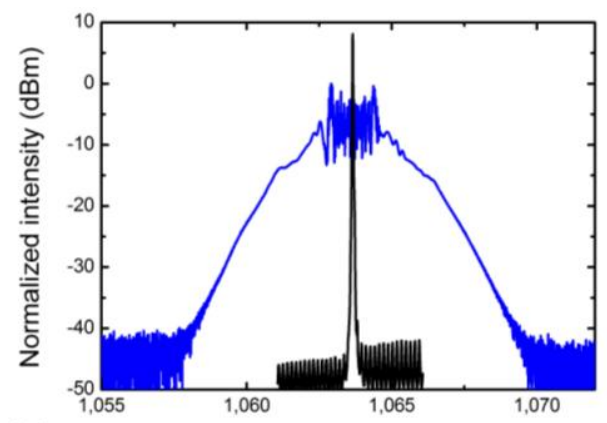

(a)

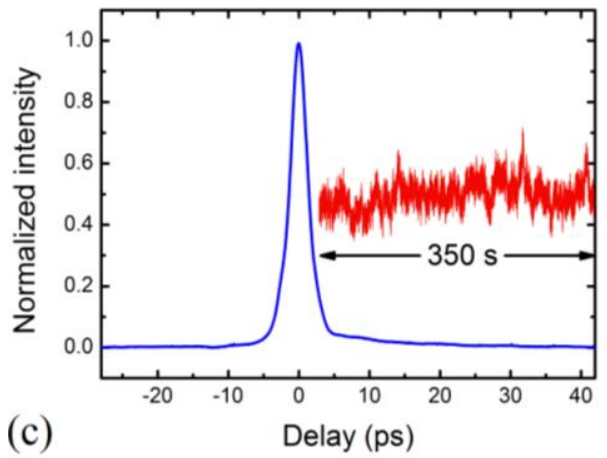

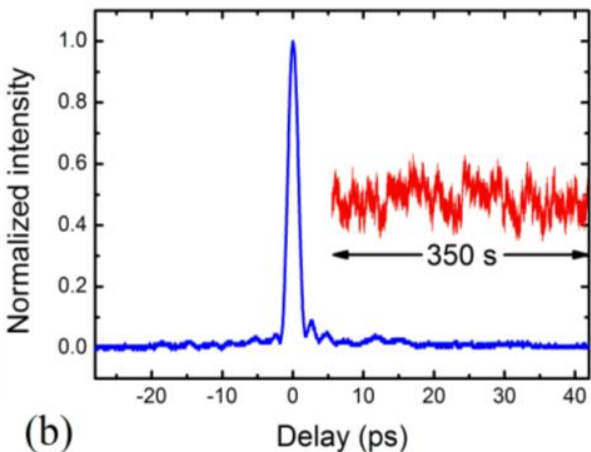

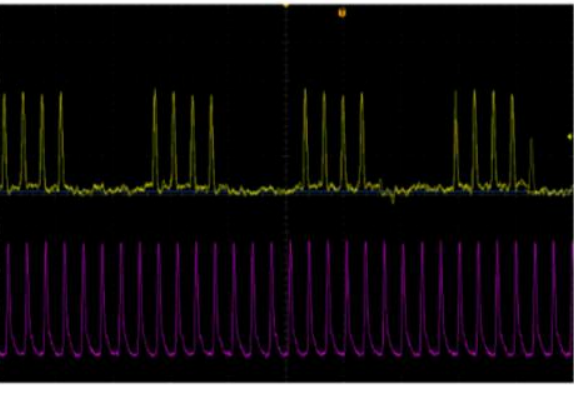

(d)

Fig. 2. Characterization of the time-lens source. (a) optical spectrum of the time-lens output (blue) and the initial input CW laser (black). (b) cross-correlation trace between the time-lens source and the 130-fs, $77-\mathrm{MHz}$ pulse train (blue) from a mode-locked fiber laser. Inset shows the measured sum-frequency signal at the half maximum of the cross-correlation trace over a time span of 350 seconds (red, same vertical scale). (c) cross-correlation trace between the time-lens source and the 2-ps, 76-MHz Ti:Sa mode-locked laser (blue). Inset shows the measured sum-frequency signal at the half maximum of the cross-correlation trace over a time span of 350 seconds (red). (d) oscilloscope trace of the time-lens source modulated by a 10MHz square wave (yellow) and the pulses of the Ti:Sa mode-locked laser (purple).

We perform label-free CRS imaging of ex vivo mouse tissues using the time-lens source. Figures 3(a) and 3(b) are SRS images taken at the $\mathrm{CH}_{2}$ stretching frequency, with the Ti:Sa laser tuned to $817 \mathrm{~nm}$. Figure 3(c) shows a single CARS image frame acquired with videorate imaging at $30 \mathrm{fps}$ (Media 1), highlighting that the time-lens source has ideal parameters for high-speed imaging. Figure 3(d) demonstrates imaging of drug delivery into mouse skin, an important application of label-free microscopy as fluorescent labeling of small drug molecules would change their penetration properties. We image the topically applied, skinactive ingredient trans-retinol at its characteristic frequency of $1600 \mathrm{~cm}^{-1}$ by tuning the Ti:Sa laser wavelength to $909 \mathrm{~nm}$. An InGaAs detector (83440C, Agilent) was used because $909 \mathrm{~nm}$ is beyond the responsive spectral range of the GaAs detector. This highlights the capability of the light-source to tune into various Raman shifts, even in the finger-print region. 

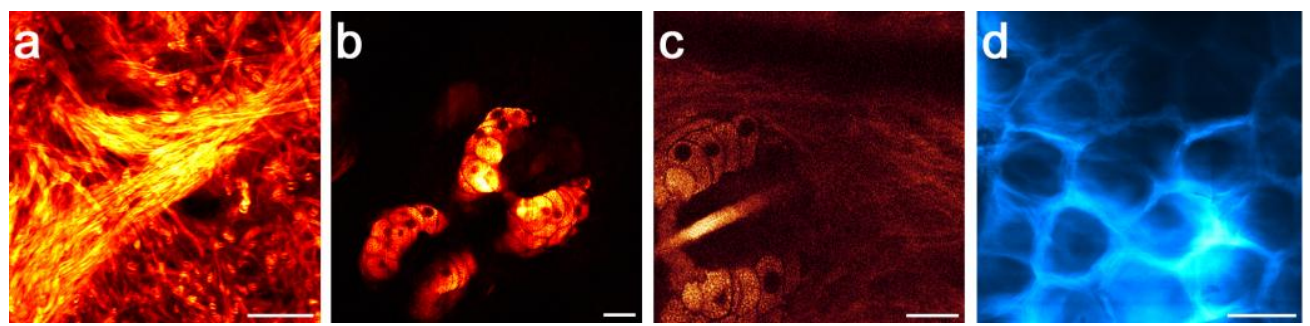

Fig. 3. Label-free CRS imaging of tissue samples. (a) SRS image of mouse brain at the $\mathrm{CH}_{2}$ stretching frequency $\left(2845 \mathrm{~cm}^{-1}\right)$, showing individual myelinated neurons. (b) SRS image of mouse skin showing the sebaceous glands with sub-cellular resolution in the viable epidermis. (c) a single frame from a CARS movie acquired at video-rate (30 fps). (d) SRS image of drug penetration of the skin-active ingredient trans-retinol in the stratum corneum obtained at the polyene stretching frequency $\left(1600 \mathrm{~cm}^{-1}\right)$. All scale bars are $25 \mu \mathrm{m}$. (Media 1)

\section{Conclusion}

The all-fiber time-lens source demonstrated here can be synchronized to any mode-locked laser, greatly increasing the flexibility for CRS imaging. When combined with a tunable CW laser that covers the gain spectrum of the fiber amplifiers, a limited amount of wavelength tunability can be provided by the time-lens source. Thus, there is a possibility for an all-fiber light source for CRS by synchronizing the time-lens source to a mode-locked fiber laser, facilitating the translation of CRS imaging from the research laboratories to the clinical setting. While we demonstrated CRS imaging, the time-lens source can be valuable to other modulation transfer techniques (e.g., stimulated emission [15], two-photon absorption [16] and excited state absorption [17]) that require the use of two synchronized light sources.

\section{Acknowledgments}

This work was supported by NIH TR01 grant 1R01EB010244-01 (to X. S. Xie) and NIH grant R01 CA133148 (to C. Xu). K. Wang, J. H. Lee, and C. Xu acknowledge the loan of Femtolite F100 laser from Prof. Chris Schaffer from Cornell University. C. W. Freudiger thanks Boehringer Ingelheim Fonds for a Ph.D. Fellowship. 